\title{
Timing of surgical decompression for traumatic cervical spinal cord injury
}

\author{
Yang Liu • Chang Gui Shi • Xin Wei Wang • Hua Jiang Chen • \\ Ce Wang • Peng Cao • Rui Gao • Xian Jun Ren • Zhuo Jing Luo • \\ Bing Wang • Jian Guang Xu • Ji Wei Tian • Wen Yuan
}

Received: 10 December 2014 / Accepted: 16 December 2014 / Published online: 11 January 2015

(C) SICOT aisbl 2015

\begin{abstract}
Purpose Although there have been numerous studies aimed at determining the effects and safety of early vs. late surgical decompression for traumatic cervical spinal cord injury, controversies still exist regarding the optimal timing of surgery for this serious spinal trauma. This study was conducted to evaluate the effectiveness and safety of early vs. late surgical decompression for lower cervical spine trauma associated with spinal cord injury.

Methods A retrospective review of was performed on consecutive patients who underwent surgical decompression for lower cervical (C3-C7) spine trauma associated with
\end{abstract}

Yang Liu and Chang Gui Shi are contributed equally to this work.

Y. Liu $\cdot$ C. G. Shi $\cdot$ X. W. Wang $\cdot$ H. J. Chen $\cdot$ C. Wang $\cdot$ P. Cao $\cdot$ R. Gao $\cdot$ W. Yuan $(\bowtie)$

Department of Orthopaedics, Changzheng Hospital, The Second

Military Medical University of China, 415 Fengyang Road,

Shanghai 200003, Peoples' Republic of China

e-mail: smmuyuanwen@163.com

\section{J. Ren}

Department of Orthopaedics, Xinqiao Hospital, The Third Military Medical University of China, Chongqing, Peoples' Republic of China

\section{Z. J. Luo}

Department of Orthopaedics, Xijing Hospital, The Fourth Military

Medical University of China, Xi'an, Peoples' Republic of China

B. Wang

Department of Orthopaedics, The Second Xiangya Hospital of

Central South University, Changsha, Peoples' Republic of China

J. G. Xu

Department of Orthopaedics, The Sixth People's Hospital, Shanghai Jiaotong University, Shanghai, Peoples' Republic of China

J. W. Tian

Department of Orthopaedics, The First People's Hospital, Shanghai Jiaotong University, Shanghai, Peoples' Republic of China spinal cord injury at six institutions across China from January 2007 to January 2012. These patients were analysed according to the timing of surgical intervention. The early group comprised patients who underwent surgery within the first 72 hours after being injured, whilst the late group comprised patients who underwent surgery after the first 72 hours. For analysis of neurologic improvement, patients who had completed a follow-up of at least six months were assessed. Other outcomes analysed were hospitalisation periods, complications and mortality.

Results A total of 595 patients were identified (456 men and 139 women at an average age of 41.4 years), with 212 in the early group and 383 in the late group. Patients in both groups had made a significant neurologic improvement in the final follow-up, but no statistically significant difference was noted between groups. Patients in the early group had a significantly shorter hospital stay ( 15.4 vs. 18.3 days, $p<0.001)$ but realised no benefits in terms of intensive care unit length of stay and ventilator days. No significant differences were identified between groups with regards complications (pneumonia, pulmonary embolism, wound infection, sepsis and urinary tract infection). Compared with the late group, the early group had a significantly higher incidence of postoperative neurological deterioration ( 6.6 vs. $0.7 \%, p<0.001)$ and mortality (7.1 vs. $2.1 \%, p=0.003)$.

Conclusion The timing of surgery for patients sustaining traumatic lower cervical spine injury with neurological involvement did not affect neurological recovery. Early surgical intervention was associated with a higher incidence of mortality and neurological deterioration compared with late surgical intervention, indicating that surgery after the first 72 hours might be relatively safe.

Keywords Cervical spine $\cdot$ Spinal cord injury $\cdot$ Surgical treatment $\cdot$ Timing 


\section{Introduction}

Spinal cord injury (SCI) is a severe traumatic disability that occurs suddenly and affects both sensory and motor functions. The estimated annual incidence of SCI worldwide varies from 10.4 per million to 83 per million [1]. SCI is an important cause of morbidity and mortality. Furthermore, the majority of patients with SCI are young, making the economic and societal impact immense [1, 2]. One third of patients with SCI experience acute traumatic cervical spine injury (fracture and/or dislocation); its impact is profound and requires systemic treatment [3, 4]. Although much improvement has been made in surgical treatment for cervical SCI and the ultimate aim could be concluded to be decompression for neural elements and reconstruction of cervical stability, controversy still exists in regards to the optimal timing of surgical intervention for this serious spinal trauma. Some authors reported that early surgery was beneficial for patients with cervical spine trauma, as the neurological condition may worsen due to haematoma formation, oedema progression and/or spinal instability [5-7]. Others found no difference between early and late decompressions with respect to neurological recovery and non-neurological outcome [8-11]. Still others argued that delaying surgery should be recommended because early surgical intervention may increase the risk of neurological function deterioration and cause iatrogenic cord injuries $[12,13]$. Therefore, the optimal timing of surgical intervention in cervical SCI has not been defined.

The purpose of this retrospective multicentre study was to compare neurological outcomes, complications and mortality in patients with traumatic cervical SCI undergoing early surgical spine (within the first 72 hours after being injured) vs. late (after the first 72 hours) intervention.

\section{Materials and methods}

This retrospective multicentre study involved six institutions throughout China. The participating investigators were asked to record information retrospectively of consecutive patients sustaining lower cervical (C3-C7) spine trauma with SCI from January 2007 to January 2012. Written informed consent to treatment and the use of clinical data for scientific purposes had been provided by all patients at the time of surgical treatment. Inclusion criteria were:

1. Traumatic cervical spinal injury between $\mathrm{C} 3$ and $\mathrm{C} 7$

2. Cervical spinal cord compression confirmed by magnetic resonance imaging (MRI), computed tomography (CT) or CT/myelography study

3. Surgical treatment, and

4. Age between 16 and 80 years.
Exclusion criteria were:

1. Combination with upper cervical spinal injury $(\mathrm{C} 1-\mathrm{C} 2)$

2. Local infection or tumour in the lower cervical spine

3. Ankylosing spondylitis.

4. Thoracic-lumbar spinal injury

5. Multisystem injury

Patients hospitalised within the first eight hours after being injured were administered methylprednisolone according to the recommendations of the Second National Acute Spinal Cord Injury Study [14]. The timing of surgical intervention was based on the time period of patients' prehospitalisation transportation, the time period of patients' medical stabilisation and the spine surgeons' preferences. Patients who underwent surgery within the first 72 hours after being injured were placed into the early group and those who underwent surgical treatment after the first 72 hours into the late group.

The authors consulted medical charts and nursing summaries. The following data were collected for each patient: gender, age, cause of injury, type of treatment, neurological status, hospitalisation periods, complications and survival. Hospitalisation periods included hospital length of stay $(\mathrm{H}-$ LOS), intensive care unit length of stay (ICU-LOS) and ventilator days (VENT DAYS). Causes of injury were categorised into four groups:

1. Road accident

2. Fall from height

3. Objects hit

4. Sports injury

Treatment approaches were classified into anterior, posterior and combination. Neurological functions were assessed before and after surgery and at the final follow-up according to the American Spinal Injury Association's modified Frankel classification as grades A-E [15]. Patients had at least a six month follow-up in terms of neurological examination, which was organised to determine the degree of neurological improvement compared with their pre-operative neurological status. The six month time period was defined for follow-up because the vast majority of neurological recovery occurs during this period [5]. Neurological improvement was defined as one grade or greater improvement according to Frankel's grading. The ratio of patients who made neurological improvement was calculated. Neurological functions were also analysed using Frankel's grade and exchanged numerically (grades A-E corresponding to scores 1-5). Patients who died or failed to undergo a six month follow-up were not analysed with respect to neurological improvement but retained in the analysis of hospitalisation periods and complications. Complications that occurred during hospitalisation were 
evaluated and included neurological deterioration, pneumonia, pulmonary embolism and infections.

Continuous variables were compared using the unpaired Student's $t$ test. Categorical data were compared using chisquare analysis. A $p$ value $<0.05$ was considered significant. All statistical analyses were performed using Statistical Product and Service Solutions Version 18.0 (SPSS, Inc., Chicago, IL, USA).

\section{Results}

Overall, 595 patients were identified (Table 1), comprising 456 men and 139 women of an average age of 41.4 (1875 ) years. Among them, 212 received early surgical and 383 late surgical decompression. There were no significant differences between groups with regard to age, gender ratio, mechanism and level of injury and type of surgical approach. The difference in the number of patients in each group who received steroids was not significant. No significant differences were identified in neurological status upon admission.

Table 1 Patients' demographic data

\begin{tabular}{|c|c|c|c|}
\hline Parameter & Early & Late & $P$ values \\
\hline Number of patients & 212 & 383 & \\
\hline Age (years) & $40.4 \pm 12.1$ & $41.9 \pm 11.5$ & 0.136 \\
\hline Male (\%) & $166(78.3)$ & $290(75.7)$ & 0.476 \\
\hline Cause of injury & & & 0.244 \\
\hline Traffic accident $(\%)$ & $121(57.1)$ & $234(61.1)$ & \\
\hline Fall $(\%)$ & $52(24.5)$ & $102(26.6)$ & \\
\hline Object hit $(\%)$ & $21(9.9)$ & $26(6.8)$ & \\
\hline Sports $(\%)$ & $18(8.5)$ & $21(5.5)$ & \\
\hline Frankel grade at admission & & & 0.300 \\
\hline A $(\%)$ & $42(19.8)$ & $82(21.4)$ & \\
\hline B $(\%)$ & $65(30.7)$ & $132(34.5)$ & \\
\hline $\mathrm{C}(\%)$ & $68(32.1)$ & $123(32.1)$ & \\
\hline $\mathrm{D}(\%)$ & $37(17.4)$ & $46(12.0)$ & \\
\hline Level of lesion & & & 0.99 \\
\hline $\mathrm{C} 3$ and/or $\mathrm{C} 3-\mathrm{C} 4$ & $16(7.5)$ & $26(6.8)$ & \\
\hline $\mathrm{C} 4$ and/or $\mathrm{C} 4-\mathrm{C} 5$ & $53(25.0)$ & $91(23.8)$ & \\
\hline $\mathrm{C} 5$ and/or $\mathrm{C} 5-\mathrm{C} 6$ & $75(35.4)$ & $141(36.8)$ & \\
\hline C6 and/or C6-C7 & $43(20.3)$ & $78(20.4)$ & \\
\hline C7 and/or C7-T1 & $25(11.8)$ & $47(12.3)$ & \\
\hline Surgical approach & & & 0.852 \\
\hline Anterior (\%) & $133(62.7)$ & $249(65.0)$ & \\
\hline Posterior (\%) & $59(27.8)$ & $101(26.4)$ & \\
\hline A-P combined $(\%)$ & $20(9.4)$ & $33(8.6)$ & \\
\hline Received methylprednisolone (\%) & $28(13.2)$ & $35(9.1)$ & 0.122 \\
\hline
\end{tabular}

$A-P$ Anterior-posterior, $C 3$ and/or $C 3-C 4 \mathrm{C} 3$ burst fracture and/or $\mathrm{C} 3-$ $\mathrm{C} 4$ dislocation
Neurological improvement was examined in patients who completed at least a six month follow-up (Table 2). Overall, 489 of the 595 patients ( 23 died and 83 failed to receive the six month follow-up) qualified for analysis, with 172 in the early and 317 in the late group. The follow-up period ranged from six to 48 months, with a mean of 24.9 months. Pre-operatively, 98 patients had complete neurological deficit (Frankel grade A), and 391 had incomplete neurological deficit (165 grade B, 169 grade $\mathrm{C}$ and 57 grade D). In the final follow-up, 106 patients $(61.6 \%)$ in the early group (Fig. 1) and 204 (64.4\%) in the late group (Fig. 2) experienced at least a one-grade improvement. No significant difference was noted in neurological improvement between the early and late groups [odds ratio $(\mathrm{OR})=0.89,95 \%$ confidence interval (CI): 0.61-1.31]. Mean preoperative Frankel grades were 2.44 and 2.34 in the early and late groups, respectively, with no statistically significant difference $(p=0.268)$. Mean postoperative scores were 3.28 and 3.26 in the early and late groups, respectively, with no statistically significant difference $(p=0.825)$. However, changes in Frankel grade from preoperatively to postoperatively were statistically significant in both the early $(p<0.001)$ and late $(p<0.001)$ groups, indicating that patients in both groups made neurological improvement after surgery.

All patients identified in this study could be analysed with respect to hospitalisation periods and complications. Table 3 displays hospitalisation periods: there was no statistical difference between groups with respect to ICU-LOS, while H-LOS was significantly longer for patients in the late group (15.4 vs. 18.3 days, $p<0.001)$. Duration of VENT DAYS, however, tended to be shorter in the late group, although this difference did not reach significance $(p=0.056)$.

Table 2 Ordinal changes in neurological outcomes ${ }^{\mathrm{a}}$

\begin{tabular}{|c|c|c|c|c|c|c|}
\hline \multirow{2}{*}{\multicolumn{2}{|c|}{ Grade at admission }} & \multicolumn{5}{|c|}{ Grade at follow-up } \\
\hline & & A & $\mathrm{B}$ & $\mathrm{C}$ & $\mathrm{D}$ & $\mathrm{E}$ \\
\hline \multicolumn{7}{|c|}{ Early surgery group } \\
\hline A & 32 & 22 & 7 & 3 & & \\
\hline B & 59 & 1 & 16 & 18 & 21 & 3 \\
\hline $\mathrm{C}$ & 54 & & 1 & 10 & 32 & 11 \\
\hline $\mathrm{D}$ & 27 & & & & 16 & 11 \\
\hline Total & 172 & 23 & 24 & 31 & 69 & 25 \\
\hline \multicolumn{7}{|c|}{ Late surgery group } \\
\hline A & 66 & 42 & 16 & 7 & 1 & \\
\hline $\mathrm{B}$ & 106 & & 28 & 32 & 39 & 7 \\
\hline $\mathrm{C}$ & 115 & & & 29 & 62 & 24 \\
\hline $\mathrm{D}$ & 30 & & & & 14 & 16 \\
\hline Total & 317 & 42 & 44 & 68 & 116 & 47 \\
\hline
\end{tabular}

Frankel grading was adopted to measure outcomes

${ }^{\text {a }}$ Patients who completed a follow-up of at least 6 months 

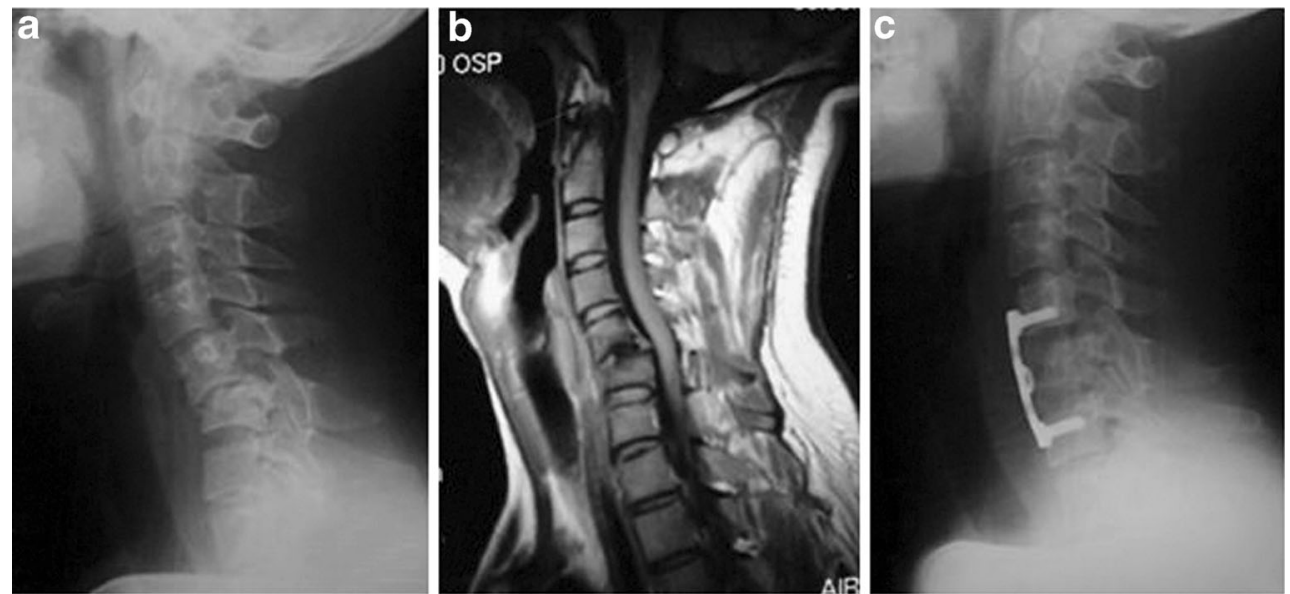

Fig. 1 A 55-year-old woman experienced severe fracture and dislocation of the lower cervical spine in a motor vehicle accident. Surgery was performed two days after injury using anterior cervical discectomy and fusion. a Pre-operative lateral X-ray reveals C5-C6 fracture and dislocation. b Magnetic resonance imaging (MRI) shows the regional kyphotic

We then examined the safety of surgical timing (Table 3). Of the 595 patients, 27 (4.5\%) experienced neurological deterioration: 18 in the early group and nine in the late group. To examine whether neurological deterioration was associated with operative intervention, these data were reanalysed with respect to deterioration before and after surgery. Overall, ten patients experienced deterioration before and 17 after surgery. Of those who deteriorated before surgery, four were in the early group and six in the late group. There was no significant difference between groups. In those who deteriorated after surgery, the incidence ( 6.6 vs. $0.7 \%$ ) was significantly higher in the early vs. the late group $(p<0.001)$. Overall, 138 deformity. c Lateral cervical spine X-ray 1.5 years postoperatively demonstrates solid fusion and good cervical sagittal alignment reconstruction. Neurological function improved from the initial Frankel grade $\mathrm{C}$ to $\mathrm{D}$ at the final follow-up; $221 \times 102 \mathrm{~mm}(300 \times 300 \mathrm{DPI})$

patients experienced pneumonia during hospitalisation: $56(26.4 \%)$ in the early group and $82(21.4 \%)$ in the late group. Although the incidence was lower in the early group, this difference was not statistically significant $(p=$ 0.166 ). There was no statistically significant difference between groups with regard to other complications, such as pulmonary embolism and infections (wound infection, sepsis and urinary tract infection).

Overall, 23 of 595 patients died during hospitalisation, with a greater in the early $(15 ; 7.1 \%)$ than in the late $(8$; $2.1 \%$ ) group, showing a statistically significant difference $(p=0.003)$. Deaths in the early group were associated with respiratory failure or cardiorespiratory causes, while
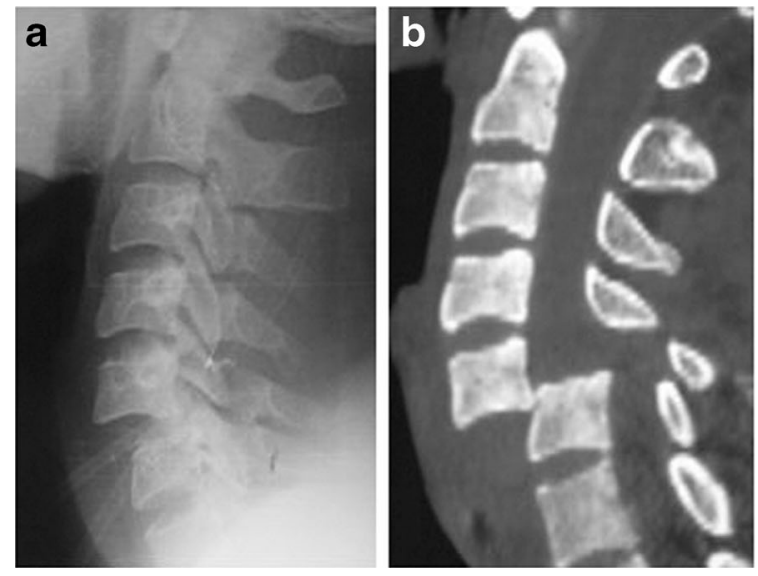

Fig. 2 A 40-year-old woman suffered severe cervical injury after a traffic accident. Surgery was performed six days after injury using posterioranterior approach and corpectomy and fixation. a Pre-operative lateral Xray revealing $\mathrm{C} 5-\mathrm{C} 6$ severe fracture and dislocation. b Threedimensional computed tomography $(\mathrm{CT})$ reconstruction showing the complete dislocated segment. c Magnetic resonance imaging (MRI)
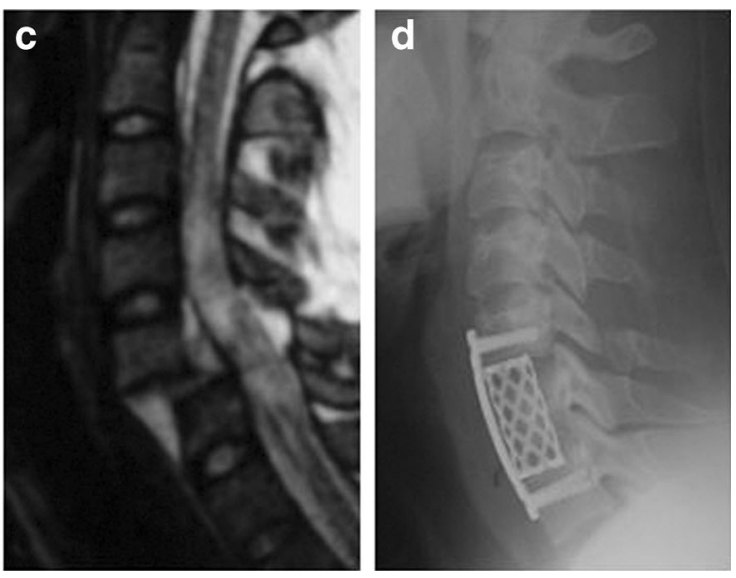

showing compressed and translated spinal cord. d Postoperative lateral cervical spine plain radiography at the latest follow-up (one year and eight months after operation) found solid fusion of certain segments and normal cervical curvature. Neurological function improved from the initial Frankel grade A to B at the follow-up; $252 \times 96 \mathrm{~mm}(300 \times 300$ DPI $)$ 
Table 3 Hospitalisation periods, complications and mortality

\begin{tabular}{llll}
\hline & Early $(n=212)$ & Late $(n=383)$ & $P$ values \\
\hline H-LOS (days) & 15.4 & 18.3 & 0.001 \\
ICU-LOS (days) & 8.1 & 8.4 & 0.150 \\
VENT DAYS & 7.3 & 7.0 & 0.056 \\
Neurologic deterioration (\%) & $18(8.5)$ & $9(2.3)$ & 0.001 \\
$\quad$ Preoperation (\%) & $4(1.9)$ & $6(1.6)$ & 0.771 \\
$\quad$ Postoperation (\%) & $14(6.6)$ & $3(0.7)$ & 0.001 \\
Other complications & & & \\
$\quad$ Pneumonia (\%) & $56(26.4)$ & $82(21.4)$ & 0.166 \\
$\quad$ Pulmonary embolism (\%) & $3(1.4)$ & $4(1.0)$ & 0.996 \\
$\quad$ Wound infection (\%) & $7(3.3)$ & $13(3.4)$ & 0.952 \\
$\quad$ Sepsis (\%) & $22(10.4)$ & $30(7.8)$ & 0.293 \\
$\quad$ Urinary tract infection & $17(8.0)$ & $42(11.0)$ & 0.249 \\
Mortality (\%) & $15(7.1 \%)$ & $8(2.1 \%)$ & 0.003 \\
\hline
\end{tabular}

$H-L O S$ hospital length of stay, ICU-LOS intensive care unit length of stay, VENT DAYS ventilator days

respiratory failure was chiefly responsible for deaths in the late group.

\section{Discussion}

Controversy over the timing of surgery for traumatic cervical spinal cord injury has existed for years. Several studies have been implemented to evaluate neurological improvement correlated with the timing of surgical intervention, but there is no consensus among them. In this study, we found no statistically significant difference between the early and late groups with respect to neurological recovery. This finding was supported by Wagner and Chehrazi [10], who reported that surgical decompression within the first 48 hours after injury had no effect on neurological recovery. In addition, Levi et al. [11] studied 103 patients and found no statistically significant difference in neurological outcomes between early and delayed groups. Tator et al. [9] found that surgical therapy did not influence neurological outcome compared with nonsurgical management. However, some other trauma surgeons reported that early surgery produced better neurological results. This effect was observed by Aebi et al. [7], who reviewed 100 patients with cervical SCI and found that immediate reduction (within the first six hours after injury) was beneficial for neurological improvement. Mirza et al. [6] analysed 43 patients from two institutions and found that patients who underwent surgery within the first 72 hours after injury showed a statistically significant neurological improvement, while those who underwent delayed surgery showed no significant change in neurological status. Results of their study must be interpreted with caution, as it compared outcomes from two different institutions. All these studies, however, were retrospective, which could hardly provide sufficient or persuasive evidence.

Two prospective trials focussed on the timing of surgery for cervical SCI. Vacarro et al. [8] evaluated the effect of early $(<72$ hours after injury) vs. late ( $>$ five days after injury) surgery on patients with cervical SCI and found no statistically significant difference in outcomes between the two groups. In another study, by Fehlings et al., (STASCIS) [5], patients who underwent early surgery ( $<24$ hours after injury) had a significantly higher percentage at least on a two-grade American Spinal Injury Impairment (AIS) Scale improvement than that of patients who underwent late surgery ( $>24$ hours after injury), while no statistically significant difference was found for a one-grade AIS improvement. The results were questioned by van Middendorp [16], who suspected that the sample size was not large enough and the analytical approach was technically incorrect. Based on raw data provided in Fehlings et al.s' study, van Middendorp calculated that there was no significant difference for a two-grade AIS improvement between the two groups. Therefore, based on the current evidence, we still could not determine the appropriate timing of surgery on neurological improvement.

Within minutes after SCI, petechial haemorrhages occur in the gray matter and oedema develops in the white matter. The haemorrhages expand during the next several hours, and oedema reaches its peak two to three days after injury, causing the level of initial cord injury to increase [17]. The extension of haematoma and oedema formed in the cervical spinal cord may lead to phrenic nerve damage or even disturb the respiratory centre. Theoretically, therefore, cervical SCI is much more serious and dangerous than thoracic and lumbar SCI. Studies show that cervical SCI was associated with a high incidence of mortality and complications when patients were analysed according to level of injury (cervical, thoracic, lumbar) $[18,19]$. Considering the high-risk conditions of the cervical spine and pathological changes of SCI in the acute stage, early surgical intervention may accelerate the progression of damage and thus cause more severe and dangerous events.

A multicentre study by Marshall et al. [12] supported the improved safety of late surgery. They enrolled 283 patients, of whom 14 had neurological deterioration. Four of the 26 patients who received surgery within the first five days after injury developed neurological deterioration; however, none of the 44 patients who underwent surgery after five days from injury experienced neurological deterioration. The authors held that early surgery for cervical SCI had a high risk of worsening spinal cord damage. Farmer et al. [20] reviewed 1,031 patients with cervical SCI, of whom 19 were identified as undergoing neurological deterioration: eight did so after surgery. Surprisingly, these eight patients underwent surgery within the first five days after injury, whereas no patients who received surgery after the first five days deteriorated postoperatively. The authors concluded that early surgery increased neurological 
morbidity. Findings regarding neurological deterioration in our study was similar to the conclusions of the two above studies $[12,20]$ that early surgery for patients with cervical SCI was associated with deterioration of neurological status.

Mortality is another safety factor that should be considered regarding surgical timing. The study we report here showed that patients who had early surgical intervention had a significantly higher incidence of mortality than those who had late surgery. Interestingly, Croce et al. [19] noted a significantly higher incidence of mortality in patients with an Injury Severity Score (ISS) $>25$ who underwent early spine fixation (within the first 72 hours after injury) than those who underwent late spine fixation. A trend towards a higher mortality was also noted in patients with cervical spine injuries. This finding was also identified by Kerwin et al. [21], who compared 158 patients undergoing spinal operative fixation within the first 48 hours after injury with 203 undergoing operative fixation after the first 48 hours. The authors noted a significantly higher incidence of mortality in patients who underwent early fixation.

Our study also emphasised nonneurological outcomes relative to the timing of surgical intervention. Patients in the early group had a significantly shorter H-LOS but realised no benefits in terms of ICU-LOS and VENT DAYS. The shorter HLOS is likely due to patients' earlier surgery and mobilisation and a greater number of early deaths. No statistically significant differences were noted with regard to complications, such as pneumonia, pulmonary embolism and infections, between groups. Although recent systematic reviews consistently concluded that early surgical treatment leads to shorter H-LOS, ICU-LOS and VENT DAYS, as well as fewer pulmonary complications [22-24], these findings should be interpreted with caution, because those reviews did not stratify or analyse cervical SCI alone. Actually, some studies focusing on patients with cervical SCI showed no statistically significant difference on these outcomes. For example, neither Kerwin et al.[18] nor Croce et al.[19] found any statistically significant differences with regard to H-LOS, ICU-LOS, VENT DAYS and incidence of pneumonia between early and late groups when patients were stratified through cervical SCI. Mirza et al.[6] noted that patients who had early surgical stabilisation ( $<72$ hours) showed no differences in minor or major complications compared with those who had stabilisation after the initial 72 hours from injury. Fehlings et al. [5] also reported that complications did not correlate with time interval from injury to decompression. Heiden et al.[13] reported an increased incidence of severe pulmonary complications in patients who had anterior surgical fusion within the first week after injury.

The time cutoffs by which to define early vs. late surgical treatment were different in various studies, mostly being 24 and 72 hours. In our study, we adopted the 72-hour cutoff due to fewer patients undergoing surgery within the first 24 hours after injury. This is likely due to the tertiary nature of referral patterns in China. Logistically, early intervention may be precluded by admitting medical facilities that lack intensive care capability and experienced personnel when faced with the need for such surgery, necessitating the patient's transfer to a specialty referral centre, thereby delaying surgery. Another reason was the poor doctor-patient relationship in China [25]. Since surgical decompression in the stage of cord oedema could worsen the possibility of iatrogenic injury, some surgeons delay the surgical time until the patient is in a relatively safe phase.

It should be noted that there are a number of limitations to the scientific design of this investigation. Its retrospective nature obviously did not allow for randomisation of patients or use of other controls to minimise patient or procedural variability. Also, surgeries were performed by different surgeons, and details of each procedure were slightly different among institutions. Variation in patient management and evaluation may account for the observed differences in results. Despite these limitations, this study offers important insights into the ongoing controversies surrounding surgical timing in cervical spin injuries.

\section{Conclusion}

We thus conclude that the timing of surgery in patients sustaining traumatic cervical spine injury with neurological deficit did not influence neurological recovery. Early surgical intervention, however, was associated with a higher incidence of mortality and neurological deterioration. Therefore, surgical decompression for cervical SCI should be delayed. When the period of stress and spinal cord oedema has passed and the patient's general condition has stabilised, surgery might be relatively safe. To determine the most appropriate timing of surgery, further studies are necessary.

\section{Conflist of interest None.}

\section{References}

1. Wyndaele M, Wyndaele JJ (2006) Incidence, prevalence and epidemiology of spinal cord injury: what learns a worldwide literature survey? Spinal Cord 44(9):523-529. doi:10.1038/sj.sc.3101893

2. Harvey C, Wilson SE, Greene CG et al (1992) New estimates of the direct costs of traumatic spinal cord injuries: results of a nationwide survey. Paraplegia 30(12):834-850. doi:10.1038/sc.1992.160

3. Sapkas G S, Papadakis S A (2007) Neurological outcome following early vs. delayed lower cervical spine surgery. Journal of orthopaedic surgery (Hong Kong). 15(2). doi

4. Trafton PG (1982) Spinal cord injuries. Surg Clin North Am 62(1): 61-72

5. Fehlings MG, Vaccaro A, Wilson JR et al (2012) Early vs. delayed decompression for traumatic cervical spinal cord injury: results of the Surgical Timing in Acute Spinal Cord Injury Study (STASCIS). PLoS One 7(2):e32037. doi:10.1371/journal.pone.0032037 
6. Mirza S K, Krengel W R, Chapman J R, et al. (1999) Early vs. delayed surgery for acute cervical spinal cord injury. Clin Orthop Relat Res. (359): 104-114. doi

7. Aebi M, Mohler J, Zach G A, et al. (1986) Indication, surgical technique, and results of 100 surgically-treated fractures and fracturedislocations of the cervical spine. Clin Orthop Relat Res. (203): 244-257. doi

8. Vaccaro AR, Daugherty RJ, Sheehan TP et al (1997) Neurologic outcome of early vs. late surgery for cervical spinal cord injury. Spine (Phila Pa 1976) 22(22):2609-2613

9. Tator CH, Duncan EG, Edmonds VE et al (1987) Comparison of surgical and conservative management in 208 patients with acute spinal cord injury. Can J Neurol Sci 14(1):60-69

10. Wagner FJ, Chehrazi B (1982) Early decompression and neurological outcome in acute cervical spinal cord injuries. J Neurosurg 56(5): 699-705. doi:10.3171/jns.1982.56.5.0699

11. Levi L, Wolf A, Rigamonti D et al (1991) Anterior decompression in cervical spine trauma: does the timing of surgery affect the outcome? Neurosurgery 29(2):216-222

12. Marshall LF, Knowlton S, Garfin SR et al (1987) Deterioration following spinal cord injury. a multicenter study. J Neurosurg 66(3): 400-404. doi:10.3171/jns.1987.66.3.0400

13. Heiden JS, Weiss MH, Rosenberg AW et al (1975) Management of cervical spinal cord trauma in southern California. J Neurosurg 43(6): 732-736. doi:10.3171/jns.1975.43.6.0732

14. Bracken MB, Shepard MJ, Collins WF et al (1990) A randomized, controlled trial of methylprednisolone or naloxone in the treatment of acute spinal-cord injury. results of the second national acute spinal cord injury study. N Engl J Med 322(20):1405-1411. doi:10.1056/ NEJM199005173222001

15. Frankel HL, Hancock DO, Hyslop G et al (1969) The value of postural reduction in the initial management of closed injuries of the spine with paraplegia and tetraplegia. I Paraplegia 7(3):179-192. doi:10.1038/sc.1969.30

16. van Middendorp J (2012) Letter to the editor regarding: "early vs. delayed decompression for traumatic cervical spinal cord injury: results of the surgical timing in acute spinal cord injury study (STAS CIS)". Spine J 12(6):540-542. doi:10.1016/j.spinee.2012.06.007

17. de la Torre JC (1981) Spinal cord injury. review of basic and applied research. Spine (Phila Pa 1976) 6(4):315-335

18. Kerwin AJ, Frykberg ER, Schinco MA et al (2005) The effect of early spine fixation on non-neurologic outcome. J Trauma 58(1): $15-21$

19. Croce MA, Bee TK, Pritchard E et al (2001) Does optimal timing for spine fracture fixation exist? Ann Surg 233(6):851-858

20. Farmer J, Vaccaro A, Albert TJ et al (1998) Neurologic deterioration after cervical spinal cord injury. J Spinal Disord 11(3):192-196

21. Kerwin AJ, Frykberg ER, Schinco MA et al (2007) The effect of early surgical treatment of traumatic spine injuries on patient mortality. J Trauma 63(6):1308-1313. doi:10.1097/TA.0b013e31815b8361

22. Carreon LY, Dimar JR (2011) Early vs. late stabilization of spine injuries: a systematic review. Spine (Phila Pa 1976) 36(11):E727E733. doi:10.1097/BRS.0b013e3181fab02f

23. Dimar JR, Carreon LY, Riina J et al (2010) Early vs. late stabilization of the spine in the polytrauma patient. Spine (Phila Pa 1976) 35(21 Suppl):S187-S192. doi:10.1097/BRS.0b013e3181f32bcd

24. Fehlings MG, Perrin RG (2005) The role and timing of early decompression for cervical spinal cord injury: update with a review of recent clinical evidence. Injury 36(Suppl 2):B13-B26. doi:10.1016/j.injury. 2005.06.011

25. Yang T, Zhang H, Shen F et al (2013) Appeal from Chinese doctors to end violence. Lancet 382(9906):1703-1704. doi:10.1016/S01406736(13)62401-0 